\title{
Violencia y agresión en el aula de matemáticas, un caso mediado por el diálogo ${ }^{1}$
}

\author{
Violence and aggression in the mathematics \\ classroom, a case mediated dialogue \\ Violência e agressividade na sala de aula de \\ matemática, um diálogo mediado caso
}

Recibido: mayo de 2013

Aceptado: agosto de 2013
Andrés Felipe Caro Moreno ${ }^{2}$

Juan Camilo Andrade Calderón ${ }^{3}$

\begin{abstract}
Resumen
En este artículo se expone una experiencia en el aula de matemáticas la cual se ve influenciada por los factores culturales y sociales de los estudiantes, la experiencia consiste en ver como un estudiante con un comportamiento hostil en el aula de matemáticas tiene en su historia cotidiana factores que afectan en su comportamiento, para ello se analizaron estos factores a partir de lo que plantean (Fernández ,2000), (Montagu, 1990), (Serrano, 1996), entre otros con el fin de determinar los factores que influyen en las acciones hostiles del estudiante dentro y fuera del aula, y una propuesta para proceder frente a los casos de agresividad y determinar su mejor solución partiendo desde la práctica y el contexto social en el aula.
\end{abstract}

Palabras clave: Aprendizaje; Aspectos afectivos; Actitud; Conflictos; Agresividad en el aula; Resolución de conflictos; Investigación e innovación en Educación Matemática; marcos teóricos; teorías de aprendizaje; situado sociocultural.

\section{Abstract}

This article presents an experience in the mathematics classroom which is influenced by cultural and social factors of the students, the experience is to see how a student with hostile behavior in the mathematics classroom has a history everyday factors that affect their behavior, for it is analyzed from these factors that pose (Fernandez, 2000), (Montagu, 1990) (Serrano, 1996), among others in order to determine the factors that influence the actions student hostile inside and outside the classroom, and a proposal to proceed against aggression cases and determine your best solution starting from the practice and the social context in the classroom.

Keywords: Learning, affective aspects, Attitude, Conflicts, Aggression in the classroom Conflict Resolution, Research and Innovation in

1 Artículo de Investigación.

2 Universidad Distrital Francisco Joe de Caldas. Bogotá, Colombia. Contacto: candres44@hotmail.com Estudiante

3 Universidad Distrital Francisco Joe de Caldas. Bogotá, Colombia. Contacto: najud_10@hotmail.com 
Mathematics Education, theoretical frameworks, learning theories, situated sociocultural.

\section{Resumo}

Este artigo apresenta uma experiência em sala de aula de matemática, que é influenciada por fatores culturais e sociais dos alunos, a experiência é ver como um aluno com comportamento hostil em sala de aula de matemática tem uma história de fatores comuns que afetam o seu comportamento, pois é analisado a partir desses fatores que representam (Fernandez, 2000), (Montagu, 1990) (Serrano, 1996), entre outros, a fim de determinar os fatores que influenciam as ações hostil aluno dentro e fora da sala de aula, e uma proposta para avançar contra os casos de agressão e determinar a melhor solução a partir da prática e do contexto social em sala de aula.

Palavras-chave: aprendizagem, aspectos afetivos, Atitude, Conflitos, Agressão em sala de aula Resolução de Conflitos, Pesquisa e Inovação em Educação Matemática, referenciais teóricos, as teorias de aprendizagem, situado sociocultural.

\section{Contextualización}

En este artículo se mostraran las acciones de Camilo en el aula escolar de matemáticas, su comportamiento y el trato que tenía con respecto a los demás estudiantes y los docentes en formación, (ESTUDIANTES PARA PROFESOR DE MATEMÁTICAS (EPPM)), dichos relatos estarán descritos desde la perspectiva de nuestra experiencia, la experiencia de la directora del curso de Camilo y la teoría referente al conflicto y la agresividad humana en el entorno social y el aula, la cual se desarrolla en el transcurso del trabajo de practica llevado por los docentes en formación.

Camilo es un estudiante de 12 años de grado quinto en la jornada mañana de un colegio que está ubicado en la localidad de Kennedy al suroccidente de Bogotá (OEA), escogimos a Camilo como protagonista de este trabajo, ya que los comportamientos de Camilo eran totalmente diferentes a los de sus demás compañeros, estos comportamientos se pueden caracterizar como:

- Agresiones verbales.

- Agresiones físicas.

- Agresiones psicológicas.
Vistas estas agresiones como el conductor entre la dominación y el reconocimiento en la escuela, evidenciando que como lo plantea Serrano (2003) "Los niños y niñas pueden aprender que la agresión es una conducta aceptable, que forma parte de la intimidad y que esa agresión o miedo es una forma correcta de ejercer control sobre los demás..." Dicha conducta agresiva procura cierto nivel de dominación frente a los demás participantes en el aula de matemáticas, la cual se observaba cuando Camilo insultaba a sus compañeros y a los docentes con palabras y actitudes hostiles.

\section{Marco Teórico}

La agresión se puede caracterizar en distintos tipos, para el estudio de la agresión de Camilo se tendrá en cuanta la agresión de tercer tipo según (Train, 2001), en la cual se evidencia que hay niños que son agresivos y dominantes al momento de hablar o expresarse en cuanto a los demás pero que no son físicamente violentos teniendo en cuenta que esta situación se encuentra fuera de un entorno de juego, como es el caso de la clase de matemáticas.

Otra de las clasificaciones que tenemos en cuenta es la agresión verbal y física en la escuela que según Serrano Sarmiento \& Iborra Marmolejo (2005) 
la agresión escolar entre los 12 y 13 años tiene los índices de violencia escolar más altos con el 26.5 $\%$. Y encontrando que el mayor nivel de agresión se genera en las aulas de clase como se observa en el gráfico elaborado por teniendo en cuenta además que otros factores inciden en las conductas agresivas.

Para determinar los factores sociales y culturales que generan que las acciones de agresión y violencia tomamos en cuenta que los"... factores de riesgo de la violencia, en particular, son variables que ponen al sujeto en una posición de vulnerabilidad hacia las conductas y actitudes violentas..." (Montagu, 1990). De las variables de vulnerabilidad tenemos que los factores familiares son:

- Prácticas de crianza inadecuadas negligentes (Pautas de crianza que se caracterizan por una ausencia de control y presencia de afecto, o bien por una falta de ambos)

- Maltrato intrafamiliar

- Familia disfuncional (esta variable se presenta cuando la familia o núcleo familiar tiene conflictos y/o problemas psicológicos)

- Poco tiempo compartido en familia. (Serrano Pintado, 1996, pág. 14)

Y a partir de esto analizar como determinar la mejor solución para las acciones de camilo en el aula de matemáticas.

\section{Descripción de la experiencia}

La agresión verbal por parte de Camilo a sus compañeros y docentes es algo común para los integrantes del aula de matemáticas, o eso se evidenciaba cuando observábamos su comportamiento en el aula, asumiendo la conducta como un acto de indiferencia por parte de los docentes y los estudiantes generando un efecto de control por parte de Camilo.

Se puede analizar que dicho hecho se encuentra inmerso en la dinámica del conflicto la cual al ejercer autoridad o poder a los demás involucrados genera que se dé la necesidad de la dominación y el reconocimiento, las cuales son comunes de los niños a esta edad según Serrano Sarmiento \& Iborra Marmolejo ( 2005) los cuales determinaron que entre los 12 y 13 años los índices de violencia escolar son los más altos con el $26.5 \%$ de ahí que se presente la actitud de dominación de Camilo dentro del aula como un factor escolar.

Por otra parte en ciertos momentos fuera del aula (descanso o receso escolar) se evidencio que Camilo golpeaba físicamente a sus compañeros tanto mujeres como hombres y finalmente ridiculizaba a los compañeros frente a los demás compañeros, mostrando una cara de satisfacción al realizar las acciones mencionadas anteriormente; los docentes en formación, al observar estas reacciones, intervinieron en el comportamiento de Camilo aunque es evidente que se presentaba lo que Montoya (2006) nombra como violencia para la dominación.

En la cual un agente de conflicto en este caso Camilo ejerce control a una población (sus compañeros) por medio del maltrato físico y psicológico (este se genera a causa de la ridiculización que hacia Camilo después de violentar), para mirar más profundamente este hecho tomamos la violencia escolar como lo plantea Serrano Sarmiento \& Iborra Marmolejo (2005) puesto que este tipo de violencia en el ámbito escolar es nombrado como Matoneo, y se presenta con mayor regularidad en el patio del colegio con el (53.4\%) por lo cual es más probable que la agresión entre estudiantes se genere en las horas del receso escolar.

La primera reacción de los docentes de la practica frente al hecho anterior fue llamar a Camilo y hablar con él (La acción la realizamos por separado cada uno de los docentes presentes).Las conversaciones que se hacían con los docentes en formación, sirvieron para establecer un vínculo de confianza entre el estudiante y los docentes de formación, en este espacio Camilo intentaba explicar las consecuencias de sus acciones en el aula, pero en ningún momento las mencionaba detalladamente, por lo tanto se llegaban a acuerdos entre el estudiante y los docentes en formación, de que Camilo iba a mejorar su comportamiento dentro y fuera de la clase de matemáticas (practicas), a estos procedimientos se les llama correctivos de lo cual los más comunes son: 
“...• En el 36,1\% de los casos, la intervención de los profesores consiste en imponer una sanción:

- Abrir un expediente (18\%).

- Expulsar de clase (6,6\%).

- Expulsar del colegio durante varios días $(6,6 \%)$.

- Según los propios alumnos que se declaran agresores, la actuación del profesorado tiene como efecto que se controlan más $(36,1 \%)$..." (Serrano Pintado, 1996)

Al transcurrir el tiempo, no se veía progreso con el compromiso que había hecho Camilo con los docentes, por lo contrario las agresiones iban incrementando cada vez más, al observar que Camilo no quería cooperar con mejorar su comportamiento en el aula de clase, dejando de la lado la sana convivencia escolar (entendida como la ausencia de violencia en un entorno determinado), hasta que sus agresiones en las demás clases llego a tal punto que los docentes de la institución buscaron a los docentes en formación para redactar un informe del comportamiento de Camilo en las clases de matemáticas (practicas), de tal forma que los directivos del colegio sancionaran a Camilo, con el fin de que tuviera un espacio de reflexión sobre el comportamiento que estaba llevando en la institución creyendo en que como se mencionó antes "....según los propios alumnos que se declaran agresores, la actuación del profesorado tiene como efecto que se controlan más $(36,1 \%) . .$. (Serrano Pintado, 1996)

En la siguiente sesión de práctica (matemáticas), los docentes en formación observamos un progreso notorio con Camilo, el cual se puede describir de la siguiente forma:

- Presentaba respeto hacia sus compañeros y docentes en formación.

- Mejoras a nivel educativo.

- Mostraba un interés en la clase.

- Reestructuro su actitud en las clases.

$\mathrm{Al}$ analizar los cambios mencionados anteriormente, los docentes en formación nos acercamos a la docente titular del curso de Camilo, es importante aclarar que esta profesora fue la única docente en la institución que no estaba de acuerdo con la sanción que le iban a colocar a Camilo, esta docente nos comentó un poco sobre la vida de Camilo, para que los docentes en formación entendieran un poco el comportamiento que tenía Camilo con las demás personas, de lo cual ella nos comenta que el núcleo familiar de Camilo estaba conformado por cinco personas incluyéndolo (mamá, papá, hermano y tía), estos miembros desempeñaban los siguientes papeles en la sociedad:

- Mamá: trabajadora sexual (Prostituta) Papá: Miembro de la cárcel (Recluso)

- Hermano: Miembro de la cárcel (Recluso)

Por lo tanto Camilo debía vivir con una tía (puesto que la mama perdió la custodia de Camilo), la cual no se preocupaba por él puesto que según la docente mencionó Camilo era una carga más para su tía, además lo que debía hacer Camilo para llegar hasta el colegio era trasladarse desde su casa ubicada a los alrededores de Alquería (Venecia al sur de Bogotá) hasta el colegio caminando porque la tía no le daba lo del bus, por tal motivo el estudiante llegaba tarde, cansado y con hambre, por lo cual era uno de los primeros en coger refrigerio ya que este era el almuerzo de Camilo.

Desde este punto de vista fue más fácil ver cuáles eran los motivos por los cuales Camilo se comportaba de manera agresiva y violenta puesto que como lo mencionan múltiples autores los factores para que se presente la violencia en el aula escolar son diversos"... factores de riesgo de la violencia, en particular, son variables que ponen al sujeto en una posición de vulnerabilidad hacia las conductas y actitudes violentas..." (Montagu, 1990)

En el caso de Camilo con la charla con la docente se evidenció que los factores familiares con los cuales podíamos relacionarlo según Serrano Pintado (1996) son:

- Prácticas de crianza inadecuadas y negligentes (Pautas de crianza que se caracterizan por una ausencia de control y presencia de afecto, o bien por una falta de ambos) 
Este factor de riesgo se evidencia en la falta de afecto por parte de la tía, puesto que la mama trabaja por la mañana en una casa como señora del servicio y por la noche como prostituta en las zonas de tolerancia de Kennedy la tía era la única que tenía contacto con Camilo, pero esta no le trataba con cariño según lo relata la docente.

- Maltrato intrafamiliar

- Además de la falta de afecto por parte de la tía esta le reprendía constantemente, lo cual se evidenciaba en rasguños y moretones en los brazos de Camilo

- Familia disfuncional (esta variable se presenta cuando la familia o núcleo familiar tiene conflictos y/o problemas psicológicos)

Al tener Camilo en su familia dos agentes endógenos inadecuados como lo son su padre y su hermano, los cuales fueron enviados a la cárcel por agresión física y a mano armada, la conducta hostil por lo cual "el desafecto, la agresión y la violencia nacen, crecen y viven en el escenario de convivencia diaria y son fruto de las relaciones interpersonales del individuo" (Fernandez, 2006)

- Poco tiempo compartido en familia.

\section{Conclusiones}

Para el tratamiento de un conflicto el cual tiene que ver con agresión y violencia en el aula de matemáticas es necesario antes de tomar cualquier decisión con respecto a las correctivas disciplinarias evaluar la situación, analizar el tipo de agresión que se presenta, como también lo son los factores de riesgo que preceden la acción de los involucrados en el conflicto para de esta forma encontrar una solución real de la problemática y no por el contrario un correctivo que en vez de ayudar a la solución total del conflicto solo lo detenga por un corto tiempo o lo alimente de tal forma que pase a circunstancias mayores.

Teniendo en cuenta lo mencionado anteriormente, la propuesta que presentamos para la solución del conflicto tiene como etapas:
Esta primera propuesta sirve para casos de conflicto bajo (discusiones, agresiones en clase, etc.), en el cual el docente puede llegar a la solución del conflicto sin recurrir a otro miembro.

- Dialogo entre los actores principales del conflicto (agresor y agredido).

- Observar los vínculos sociales del agresor (familiares, entorno).

- Análisis del entorno donde se generó el conflicto.

- Dialogo entre personas allegadas a los actores o involucrados en el conflicto.

- Búsqueda de pruebas que ratifiquen las versiones obtenidas de los diálogos (testigos).

- Toma del respectivo correctivo referente al conflicto.

- Análisis de las consecuencias del correctivo.

- Valoración del caso.

- Intervención del maestro para la solución del conflicto (maestro se comunica con cada uno de los miembros del conflicto y realiza un compromiso de no agresión entre ellos y con ningún otro compañero).

\section{Referencias}

Fernandez, I. (2006). Escuela sin violencia. Mexico: Alfaomega Grupo Editor.

Montagu, A. (1990). La naturaleza de la agresividad humana. Alianza.

Montoya, V. (2006). Teorias de la violencia humana. Redalyc , 1-8.

Serrano Pintado, I. (1996). Agresividad infantil. Madrid: Pirámide.

Serrano Sarmiento, Á., \& Iborra Marmolejo, I. (2005). Violencia entre compañeros en la escuela. Barcelona: Diceñarte.

Train, A. (2001). Agresividad en niños y niñas. Narcea Editores. 